\title{
Use of $\beta$-Cyclodextrins to Prevent Modifications of the Properties of Carbopol Hydrogels Due to Carbopol-Drug Interactions
}

\author{
Haydee Blanco-Fuente, Blanca Esteban-Fernández, José Blanco-Méndez, and \\ Francisco-Javier OTERo-EsPINAR*
}

Department of Pharmacy and Pharmaceutical Technology, Faculty of Pharmacy, 15706 Santiago de Compostela. Spain. Received June 25, 2001; accepted October 5, 2001

\begin{abstract}
Carbomers are carboxyvinylic derivatives that are widely used in the manufacture of hydrogel dosage forms. Because of their anionic nature and large number of acid groups, they tend to interact with cationic substances, and with other hydrophilic polymers containing alcohol groups. Here, we report a study of interactions between the carbomer $\mathrm{Carbopol}^{\circledR}$ and the cationic drug propranolol hydrochloride in the solid state and in solution, and of the effects of such interactions on the properties of the hydrogel. We found that the drug forms an insoluble ionic complex with the polymer, modifying all of the hydrogel properties studied (swelling, release, bioadhesion). The inclusion of $\beta$-cyclodextrin in the formulation reduces polymer/drug interactions, so that hydrogel properties remain unchanged. This is probably attributable to formation of inclusion complexes of $\beta$-cyclodextrin and the drug, so that the drug is prevented from interacting with the polymer.
\end{abstract}

Key words hydrogel; carbomer; $\beta$-cyclodextrin; propranolol hydrochloride; polymer-drug interaction; bioadhesion

Hydrogels are networks constituted by a crosslinked polymeric material that swells in water and biological fluids. The characteristics of hydrogels make them highly suited for use in controlled-release systems, whether at swelling equilibrium or as part of dynamic systems in which release and swelling occur simultaneously. ${ }^{1}$ Numerous studies have investigated the behaviour of a) hydrogels that swell in response to ambient conditions $(\mathrm{pH}$, temperature, ionic strength, concentration of chemicals), ${ }^{2,3)}$ and b) of bioadhesive hydrogels. ${ }^{47)}$ Most such systems are constituted by hydrophilic polymers containing a large number of ionizable groups (carboxyls, hydroxyls, amines, etc.), notably metacrylic acid and polyacrylic acid derivatives.

A major drawback of hydrogel-forming polymers is that they typically interact with many types of molecule, such as other polymers or low-molecular-weight molecules including certain drugs. $^{8-10)}$ The nature of such interactions varies (ionic interaction, hydrogen bonds, etc.), depending on the characteristics of the polymer and the drug.

The permeability of solutes through hydrogels depends largely on the hydrogel's internal structure, which governs its porosity, hydrophobicity, elasticity and thus swelling and release properties. When interactions arise between a hydrogelforming polymer and another component of the formulation, the properties of the formulation may be affected, with consequent effects on both its efficacy and stability. The elimination or minimization of interactions of this type is thus clearly of great practical interest.

Cyclodextrins (CDs) are pharmaceutical excipients frequently used with the aim of increase in the solubility and bioavailability of poorly hydrosoluble drugs. One of the key advantages of $\mathrm{CD}$ inclusion compounds is that the drug is contained within the $\mathrm{CD}$ cavity and thus protected from the external medium. Using this approach, labile drugs have been protected against oxidation, hydrolysis and photodecomposition. ${ }^{11)}$ Furthermore, the complexation of drug molecules in the apolar interior of $\mathrm{CD}$ inclusion compounds may induce changes in drug properties including $\mathrm{p} K_{\mathrm{a}}$, spectroscopic properties (visible/UV absorption, IR absorption, or fluorescence) and chemical properties (notably reactivity).

In the present study we investigated interactions between three varieties of $\mathrm{Carbopol}^{\circledR}$ (a carbomer that is widely used in the manufacture of pharmaceutical gels and bioadhesive hydrogels) and propranolol hydrochloride (PRP, a cationic drug). The Carbopols are high-molecular-weight carboxyvinylic derivatives comprising polyacrylic acid subunits crosslinked with allyl saccharose or pentaerithritol allyl ethers. PRP is known to interact with polyacrylic acid, ${ }^{10)}$ and to form stable inclusion compounds with CDs (binding constant of $220 \pm 20 \mathrm{~m}^{-1}$ at $\mathrm{pH} 7$ calculated using liquid chromatography method). ${ }^{12)}$ We investigated the effects of carbomer/drug interactions on the principal characteristics of the system, namely drug solubility, swelling, drug release and bioadhesiveness. We also investigated the capacity of a $\beta$ - $\mathrm{CD}(\beta \mathrm{CD})$ to inhibit the carbomer/drug interactions.

\section{Experimental}

Pharmaceutical Materials The three Carbopol varieties used (Carbopol $^{\circledR} 941,934,940$ ) were a gift from B. F. Goodrich (France). The three varieties differ in degree of crosslinking, and thus in viscosity and molecular weight: crosslinking is highest in Carbopol 940 and lowest in Carbopol 941. PRP hydrochloride was from Roig Farma (Spain). PRP base was obtained by neutralization of a solution of PRP hydrochloride with sodium hydroxide; the precipitate obtained was washed repeatedly with deionized water and dried to constant weight at $50^{\circ} \mathrm{C} . \beta \mathrm{CD}$ (Kleptose ${ }^{\circledR}$, Roquette) was a gift from Laisa (Spain). All reagents used were analytical grade.

Study of Polymer/Drug Interactions in Solution Solutions containing Carbopol 941 and PRP (hydrochloride or base) at various proportions were obtained by mixing a solution of the polymer and of drug of different concentrations. Insoluble species were detected by turbidimetry at $350 \mathrm{~nm}$, in a Hewlett-Packard 8452A diode-array spectrophotometer (Germany), and separated out by filtration and subsequent drying to constant weight at $50^{\circ} \mathrm{C}$. The complexes were then analysed by (IR) spectroscopy in a Mattson Cygnus 100 fourier transform (FT)-IR spectrometer using $\mathrm{KBr}$ discs. Kinetic studies and inclusion-complex-formation isotherms were done using Visking membranes (cellulose based tubes of $1350 \mathrm{MWCO}$, Medicell International Ltd.) containing $5 \mathrm{ml}$ of a $0.1 \mathrm{~g} / 1$ solution of Carbopol. The bags were immersed in vials containing $15 \mathrm{ml}$ of a solution of PRP hydrochloride $(40 \mathrm{mg}$ in $15 \mathrm{ml}$ for the kinetic studies, various concentrations for isotherms). At predetermined time intervals over a $24 \mathrm{~h}$ period (or after $24 \mathrm{~h}$ for isotherms), samples of the drug solution were removed for determination of drug content by UV spectrometry at $288 \mathrm{~nm}$. All assays were performed in deionized water at $37^{\circ} \mathrm{C}$ and was realised in triplicate. 
To investigate the influence of $\beta \mathrm{CD}$ on the formation of the precipitate, equivalent assays were performed after introduction of different amounts of $\beta \mathrm{CD}$ (5-30\% of polymer weight) into the dialysis bag with the polymer, in all cases with a polymer concentration of $0.1 \mathrm{~g} / 1$ and a PRP hydrochloride concentration of $0.75 \mathrm{~g} / 1$.

Preparation and Evaluation of Bioadhesive Hydrogels Carbomer xerogels containing PRP hydrochloride and $\beta \mathrm{CD}$ were prepared by the compression/heating method of Blanco-Fuente et al. ${ }^{13)}$ Briefly, $200 \mathrm{mg}$ of Carbopol or a Carbopol $-\beta \mathrm{CD}$ mixture ( 5 or $20 \% \beta \mathrm{CD}$ by weight) and $50 \mathrm{mg}$ of PRP hydrochloride were mixed, then compressed in a $13-\mathrm{mm}$-diameter matrix under $7500 \mathrm{~kg}$ for $2 \mathrm{~min}$, then cured at 90,110 or $130^{\circ} \mathrm{C}$ for $4 \mathrm{~h}$.

Hydrogel swelling was evaluated by placing the xerogel in a recipient containing $100 \mathrm{ml}$ of phosphate buffer (pH 7.2). Diameter and thickness were measured at predetermined time intervals, and after $24 \mathrm{~h}$ the hydrogel was removed from the buffer, carefully padded dry and then weighed to determine amount of water absorbed at equilibrium. To compare the swelling of the different formulations, we determined a) area-under-the-curve $(A U C)$ in graphs of hydrogel volume against time $(0-24 \mathrm{~h})$, calculated by the trapezoidal rule using the program Microcal Origin ${ }^{\mathrm{TM}}$ version 6.0 (Microcal Software, Inc., U.S.A.), b) equilibrium aqueous volume $(E A V)$ as $V_{24} / V_{0}$ where $V_{24}$ is volume after $24 \mathrm{~h}$ and $V_{0}$ is initial volume, and c) equilibrium water content $(E W C)$ as $Q_{24} / Q_{0}$ where $Q_{24}$ is water content after $24 \mathrm{~h}$ and $Q_{0}$ is initial water content.

Release assays were performed in a Hewlett Packard automatic dissolution testing connected to a Prolabo Dissolutest apparatus (method no. 2, USP 23rd Ed.). The dissolution medium was a litre of phosphate buffer ( $\mathrm{pH}$ 7.2). The xerogels were placed in nylon-mesh baskets $(0.05 \mathrm{~mm}$ mesh size), sufficiently large to allow unimpeded hydrogel swelling, to prevent them from floating to the surface.

The hydrogels tested were prepared with 100,150 or $200 \mathrm{mg}$ of Carbopol, $50 \mathrm{mg}$ of PRP hydrochloride, and in some cases 5 or $20 \%$ (with respect to polymer weight) of $\beta \mathrm{CD}$.

For comparison of drug release behaviour among formulations, we determined $A U C$ of graphs of percentage drug released against time $(0-8 \mathrm{~h})$, and percentage drug released after 2 and $8 \mathrm{~h}$.

Alterations in the in vitro adhesive capacity of hydrogels were studied by tension methods, ${ }^{14)}$ using a Lloyd LR5K tension tester (Lloyd, U.K.), with a $50 \mathrm{~N}$ load cell. Assay were conducted as detailed in Blanco-Fuente et al. ${ }^{13,15)}$ Adhesion assays were done under two sets of hydration conditions. In the first, $25 \mu$ l of water was spread uniformly over the surface of the hydrogel (limited hydration condition (LHC) assays). In the second, the entire assay system (hydrogel and substrate) was immersed in a water bath thermostatted to $37^{\circ} \mathrm{C}$, allowing unrestricted swelling of the hydrogel (nonlimited hydration condition (NLHC) assays). In both cases an initial force of $0.5 \mathrm{~N}$ was applied for $20 \mathrm{~min}$, and then traction at $5 \mathrm{~mm} / \mathrm{min}$. For evaluation of results, work of adhesion and Young's moduli was estimated from the force-elongation curves obtained during the traction phase.
Statistical Analyses Between-treatment comparisons were done by analysis of variance, following confirmation of data normality and homogeneity of variances. Subsequent pairwise comparisons were done by the Student-Newman-Keuls test. Unless otherwise stated, statistical significance is taken to be indicated by $p<0.01$. All statistical analyses were performed with Statistica, Statsoft Inc. Experimental data were fitted to theoretical models using the nonlinear least-squares fitter contained in the program Microcal Origin 6.0. The preliminary estimates required for this procedure were obtained by linear regression following linearization of the data.

\section{Results and Discussion}

Polymer/Drug Interactions When the drug was dissolved in a dispersion of the polymer, a whitish, scarcely soluble, slowly precipitating product was immediately formed. This product gives rise to an increase in the turbidity of the system, which depends on the quantity of precipitate formed and can thus be used to evaluate the intensity of the polymer/drug interaction. Figure 1 shows the turbidity values obtained with mixtures containing different proportions of Carbopol 941 and PRP. The quantity of precipitate increases with increasing amount of drug. This phenomenon is observed whether the drug is in hydrochloride or base form; in polymer dispersions with higher polymer concentration, however, the increase was greater when the drug was in base form. The difference between hydrochloride and base is not attributable to a more intense interaction with the latter, but rather to the marked increase in the dispersion viscosity caused by the base, impeding flocculation and sedimentation of the precipitate. The increase in viscosity is attributable to neutralization of the Carbopol.

To investigate the nature of the interactions between the two components, we isolated the polymer/drug coprecipitate and analysed it by FT-IR spectroscopy (Fig. 2). The Carbopol spectrum shows a $\mathrm{C}=\mathrm{O}$ stretching band at about $1714 \mathrm{~cm}^{-1}$, corresponding to the numerous carboxylic acids present in the chain. The wavelength at which this peak occurs indicates that the carboxyl groups are associated via hydrogen bonds, forming intra- or intermolecular dimers. Ionization of these groups by the addition of sodium hydroxide leads to breakage of the hydrogen bonds which induces a reorganiza-

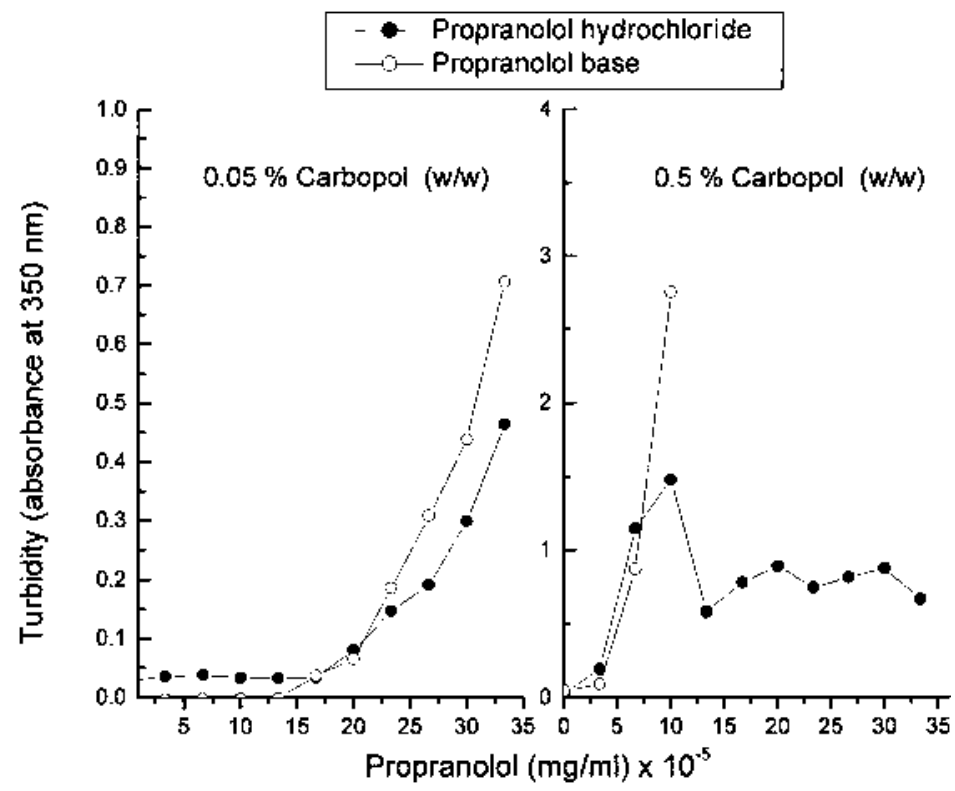

Fig. 1. Turbidity at $350 \mathrm{~nm}$ of 0.05 and $0.5 \%$ Solutions of Carbopol 941, in the Presence of Increasing Concentrations of PRP Hydrochloride or PRP Base 


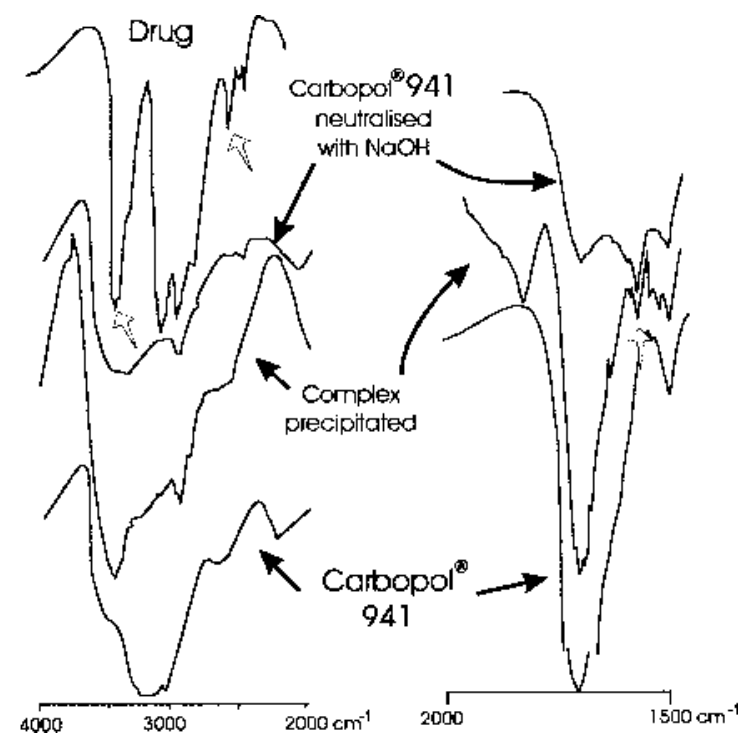

Fig. 2. FT-IR Spectra of Carbopol 941, PRP Hydrochloride, Carbopol 941 Neutralized with $\mathrm{NaOH}$, and the PRP/Carbopol Complex

Arrows indicate the most significant bands.

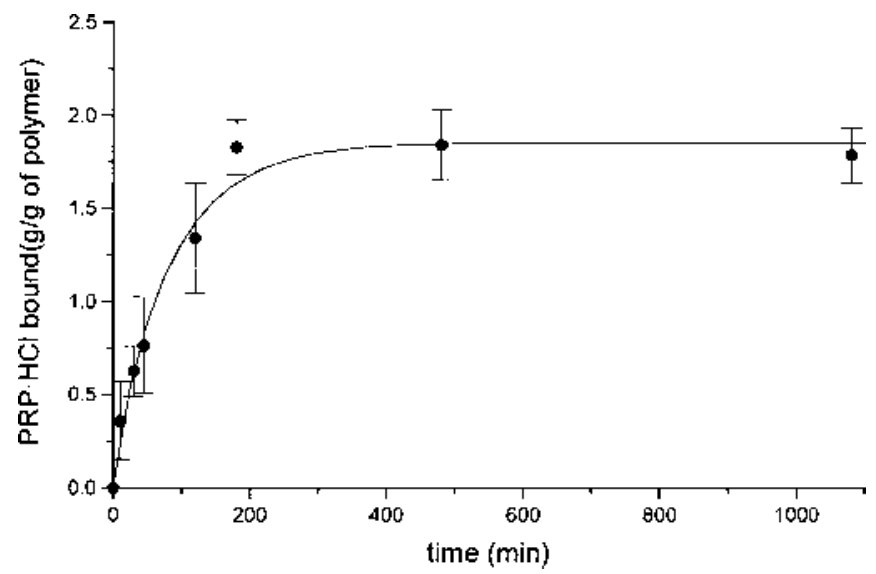

Fig. 3. Rate of Formation of the Ionic Complex between Carbopol 940 and PRP Hydrochloride

tion of the electron clouds of the carbonyl groups, shifting the stretching band to $1579 \mathrm{~cm}^{-1}$, characteristic of ionized groups. The spectrum for the isolated drug-Carbopol coprecipitate shows both bands, at 1714 and $1579 \mathrm{~cm}^{-1}$. This indicates that the precipitate contains both ionized and non-ionized carboxyl groups forming dimers, suggesting that the drug causes partial ionization of the polymer. This is supported by the fact that the coprecipitate spectrum does not show the characteristic hydrochloride band observed in the $2700-2250 \mathrm{~cm}^{-1}$ region in the PRP hydrochloride spectrum.

Having characterized the polymer/drug interaction, we next studied the rate of formation of the insoluble product, and the influence of polymer molecular weight on this interaction. As can be seen from Fig. 3, the interactions occur rapidly, although more than $5 \mathrm{~h}$ are necessary for equilibrium to be reached. The amount of PRP hydrochloride bound to the polymer at equilibrium was $1.85 \mathrm{~g} / \mathrm{g}$, about $30 \%$ of the amount of drug in solution.

To determine the maximum amount of drug interacting with the polymer, we constructed isotherms for the formation

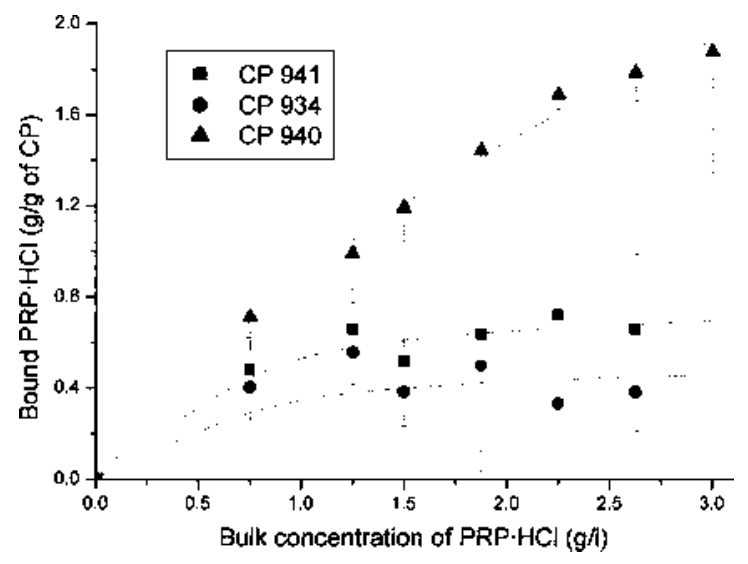

Fig. 4. Formation Isotherms for the Carbopol/PRP Ionic Complex

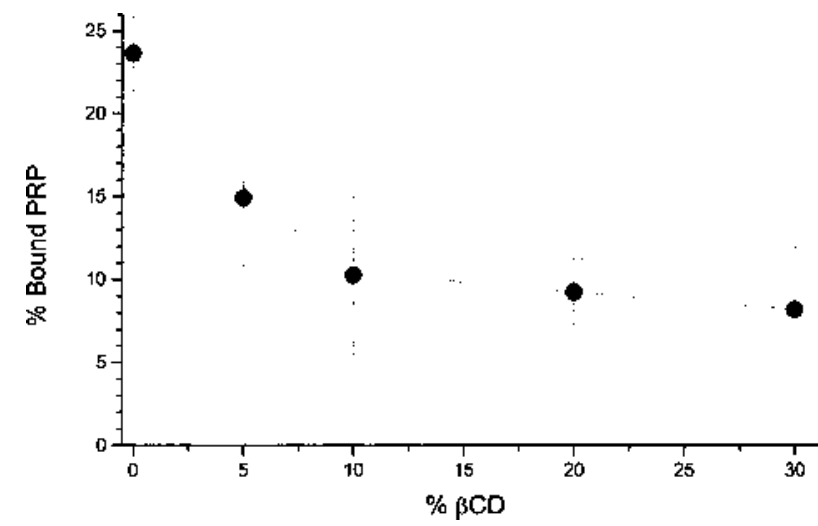

Fig. 5. Influence of $\beta C D$ on the Formation of the Carbopol/PRP Ionic Complex

All assays were performed with $0.1 \mathrm{~g} / 1$ of Carbopol 940 and $0.1 \mathrm{~g} / \mathrm{l}$ of drug.

of polymer/drug complex with the three Carbopol varieties (Fig. 4). In all cases, the curves were Langmuirian, indicating that complex formation is limited by the scarcity of drugbinding regions on the polymer chain. The points of union, as determined by FT-IR, are constituted by the numerous acid groups of the polymer chain. The saturation value could be calculated using the Langmuir model. ${ }^{16)}$ The estimated plateau-level concentrations of PRP hydrochloride $(\mathrm{g} / \mathrm{g}$ of polymer) were $0.80 \pm 0.11,0.54 \pm 0.20$ and $4.6 \pm 0.60$ for Carbopol 941, 934 and 940 respectively. The number of free acid groups per gram of polymer is similar in the three varieties, with only minor differences due to crosslinking. The variations in amount of drug bound may thus be due to the differences in molecular weight and degree of crosslinking between the three varieties of polymer; these differences undoubtedly affect the spatial structure of the hydrated polymer, which in turn influences the availability of free acid groups, polymer solubility and hydration.

To confirm the possible efficacy of CDs as inhibitors of polymer/drug interactions, we determined amount of drug bound in assays in which different amounts of $\beta C D$ were included with the polymer/drug mixture. The results obtained (Fig. 5) show that increasing concentrations of $\beta C D$ led to decreased amounts of drug bound. The maximum effect is obtained at a $\beta C D$ concentration of $10 \%$, with higher concentrations having no additional effect.

Both the establishment of polymer/drug interactions and 


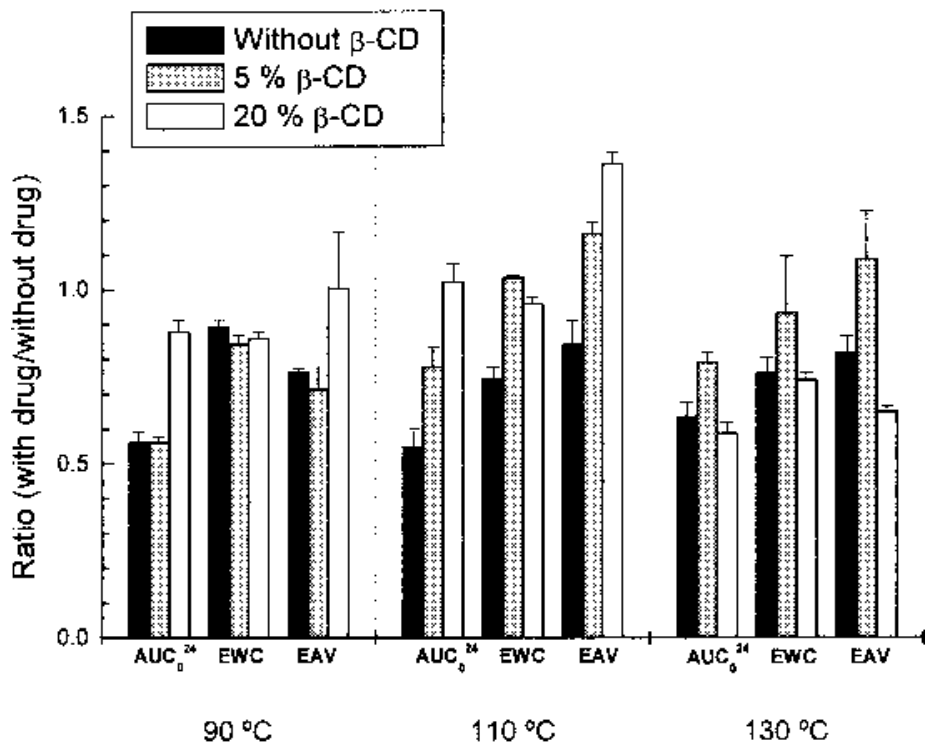

Fig. 6. Influence of $\beta C D$ on Swelling of Carbopol 940 Hydrogels

$A U C_{0}^{24 h}$ is the $A U C$ of the plot of xerogel volume against time, first $24 \mathrm{~h} ; E A V$ and; $E W C$; see Materials and Methods.

inclusion complex formation are reversible processes, the intensity of the interaction being described by a stability constant. The inhibitory action of $\beta \mathrm{CD}$ suggests that it competes with the polymer for the drug, probably because the drug is able to form highly stable inclusion complexes with the $\beta \mathrm{CD}$ (CITA/S), with stability constants higher than those of polymer/drug complexes.

Having characterized polymer/drug interactions in dissolution, we next investigated whether these interactions affect the principal properties of formulations prepared with these products, and if so whether CDs are able to inhibit these effects.

Evaluation of Bioadhesive Hydrogels Hydrogel hydration and swelling are of key importance, since they are major determinants of properties including drug release rate and bioadhesiveness. Hydration and swelling depend above all on the balance of forces between solvent and polymer, the most important polymer properties being degree of crosslinking, and abundance and nature of hydrophilic sites in the macromolecular matrix. Any modification of polymer-polymer or polymer-solvent affinity will have a direct influence on the elastic tension that controls swelling. Figure 6 summarizes the modifications in swelling parameters $\left(A U C_{0}^{24 \mathrm{~h}}, E A V\right.$, $E W C$ ) induced by incorporation of 5 or $20 \% \beta C D$ into Carbopol 940 hydrogels. In all cases $(0,5$ or $20 \% \beta \mathrm{CD}$; all crosslinking temperatures of $90-130^{\circ} \mathrm{C}$ ), inclusion of the drug in the hydrogel reduced all three swelling parameters by about $50 \%$. The formation of an ionic polymer/drug complex leads to insolubilization and folding of the polymer chains, reducing affinity for the solvent and increasing elastic tension.

In some of the hydrogels prepared without drug, the inclusion of $20 \% \beta \mathrm{CD}$ slightly reduced swelling (though in all cases the values observed were much higher than in hydrogels prepared with drug). This is attributable both to the reduced proportion of polymer in the hydrogel $(80 \%)$ and to reduced system mass as a result of solubilization of $\beta C D$.

In hydrogels prepared with drug, by contrast, the inclusion of $\beta \mathrm{CD}$ led to increased swelling, in general to levels similar to those observed in hydrogels without drug and with $\beta \mathrm{CD}$.

The results indicate that, during the hydration and swelling process, $\beta \mathrm{CD}$ is capable of forming complexes in situ with PRP hydrochloride, preventing insolubilization of the polymer and maintaining the swelling characteristics of the hydrogel. It is of interest that this can be achieved by simply including the $\beta \mathrm{CD}$ during polymer/drug complex formation, there being no need to mix the $\beta \mathrm{CD}$ and the drug previously.

Since hydration and swelling are essential for drug release from hydrogels of this type, any modifications in hydration/ swelling properties can be expected to affect release rate. Since it is of course impossible to determine release from hydrogels without drug, we compared release from hydrogels containing drug and different proportions of polymer and $\beta C D$. Figure 7 shows the release profiles obtained for xerogels prepared with $200 \mathrm{mg}$ of Carbopol 940. Fitting of the nonlinear equation proposed by Peppas and Korsmeyer ${ }^{1)}$ for flat dosage forms,

$$
M_{t} / M_{\infty}=k t^{n}
$$

permits assessment of the mechanisms by which the drug is released to the medium. $M_{\infty}$ and $M_{t}$ are the amount of drug incorporated in the xerogels and the drug released at time $t$ respectively, $k$ and $n$ are system parameters that depend of the nature of polymer-drug-solvent interaction.

The values of between 0.66 and 0.92 obtained for the coefficient $n$ ( $r$ between 0.9904 and 0.9999) indicate that in all cases release occurs as a result of the "anomalous transport" mechanism, in which release is controlled by polymer relaxation and swelling, and subsequent diffusion of the drug through the gel. We did not detect significant differences between the coefficients obtained with hydrogels containing different proportions of $\beta \mathrm{CD}$, indicating that despite inhibition of the formation of the polymer-drug complex, $\beta \mathrm{CD}$ affected only the rate of release, not its mechanism.

Elimination of the first and last sections of the release profiles ( $\%$ drug release $<10 \%$ and $>70 \%$ ) enabled good fits to 

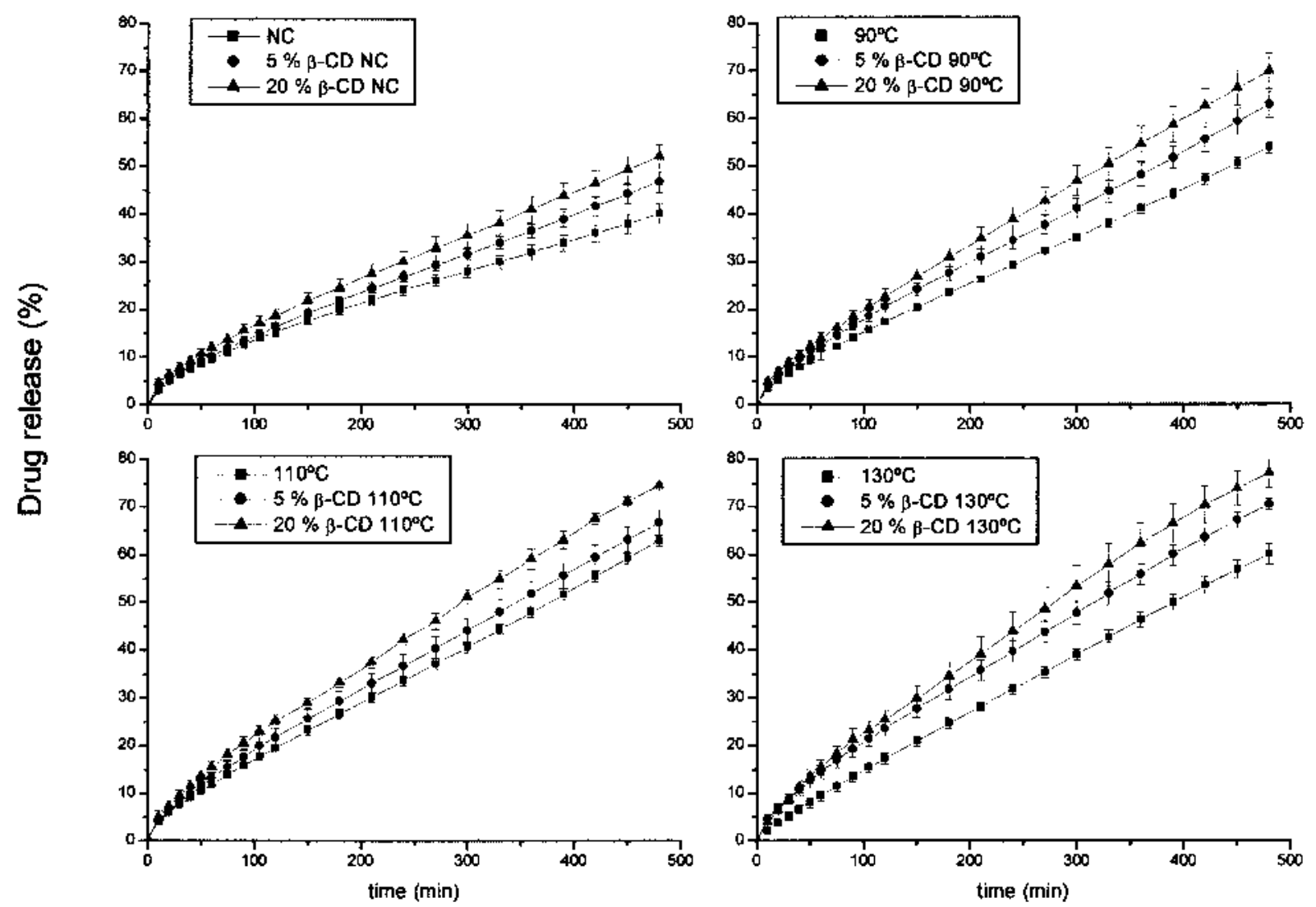

Fig. 7. Drug-Release Profiles for Carbopol 940 Xerogels Prepared with Different $\beta C D$ Concentrations $(0,5$ or $25 \%)$ and at Different Crosslinking Temperatures $\left(90,110\right.$ or $130^{\circ} \mathrm{C}$, or No Crosslinking (NC))

be obtained with zero-order release equations $(r$ between 0.9950 and 0.9999$)$. The best fits were obtained with the hydrogels containing the highest proportion of polymer. Again, the good fit of this model indicates that polymer relaxation, polymer swelling and drug diffusion are important determinants of the release profile. The rate constants and $A U C_{0}^{8 \mathrm{~h}}$ values obtained are shown for the different formulations tested in Fig. 8. The observed behaviour is in accordance with the modifications detected in swelling and release mechanism. In all cases the inclusion of $\beta C D$ led to a moderate increase in release rate and amount of drug released, although in no case was this increase spectacular.

Of the polymers used as excipients in pharmaceutical technology, Carbopol is one of the most strongly mucoadhesive, and is thus frequently used in bioadhesive formulations. The mucoadhesive capacity of this polymer is influenced by various factors related to its structure and to the composition of the fluid present at the site of adhesion. ${ }^{14,16-18)}$ As a result, anything that modifies the physicochemical characteristics of the polymer or its affinity for the hydration medium will tend to alter its bioadhesive capacity. Before adhesive bonds form between the polymer and the biological substrate, the chains of the polymer must hydrate, giving the polymer sufficient freedom to diffuse into the interior of the biological substrate. Furthermore, hydration gives the hydrogel an elastic structure that affects the strength of the bioadhesive forces. It is therefore to be expected that the adhesive capacity of hydrogels containing PRP hydrochloride will be modified by polymer/drug interactions. The force-elongation curves ob- tained in the adhesion assays under LHC (Fig. 9) confirm these assumptions. The inclusion of the drug clearly increased the elasticity of the polymer in the presence of small quantities of water, leading to a marked increase in the degree to which the hydrogel was deformed before breakage of polymer/substrate adhesive bonds. In addition, the force-elongation curves for drug-containing hydrogels showed a characteristic "saw-tooth plateau," indicating that polymer/substrate separation did not occur cleanly and instantaneously, but irregularly over the bound surface. Young's moduli for hydrogels with and without drug (Table 1) confirm the increase in elasticity induced by the drug. Work of adhesion is the sum of i) a thermodynamically reversible component that is dependent on the type and intensity of the adhesive bond, and ii) an irreversible component related to deformations of the polymer system and dependent on its viscoelastic properties. ${ }^{14)}$ In the majority of cases, the irreversible component is several orders of magnitude higher than the reversible component, and is thus of much greater real importance.

However, markedly different results were obtained in adhesion assays under NLHC. In such systems, the work of adhesion of drug-containing hydrogels is lower, since the irreversible component is lower. At high levels of hydration, water acts as a plasticizer, reducing Young's modulus in all the hydrogels, so that elasticity scarcely varies when the drug is included. Under these conditions, the mobility and diffusivity of the polymer chains, together with substrate affinity, acquire greater importance than adhesion differences. 


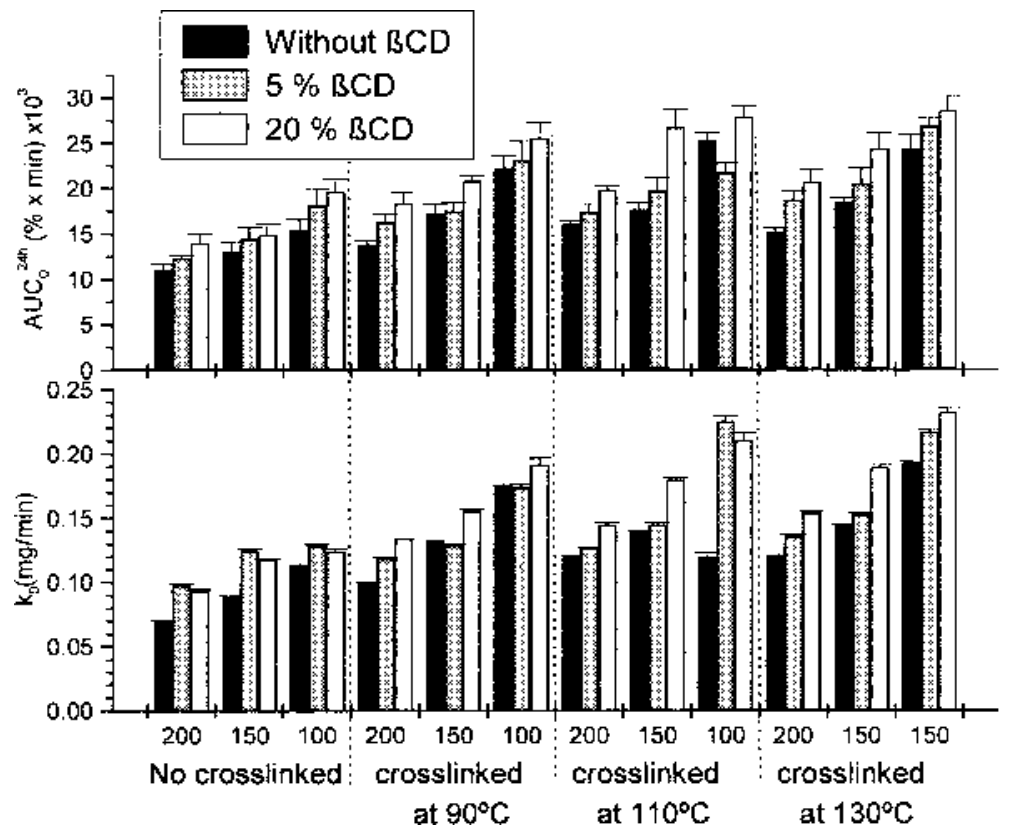

Fig. 8. Estimated Values of the Rate Constant $k_{0}$ and the Parameter $A U C_{8 \mathrm{~h}}$, for Dissolution of PRP Hydrochloride from Carbopol 940 Xerogels Prepared with Different $\beta$ CD Concentrations ( 0,5 or $25 \%)$ and at Different Crosslinking Temperatures $\left(90,110\right.$ or $130^{\circ} \mathrm{C}$, or No Crosslinking (NC))

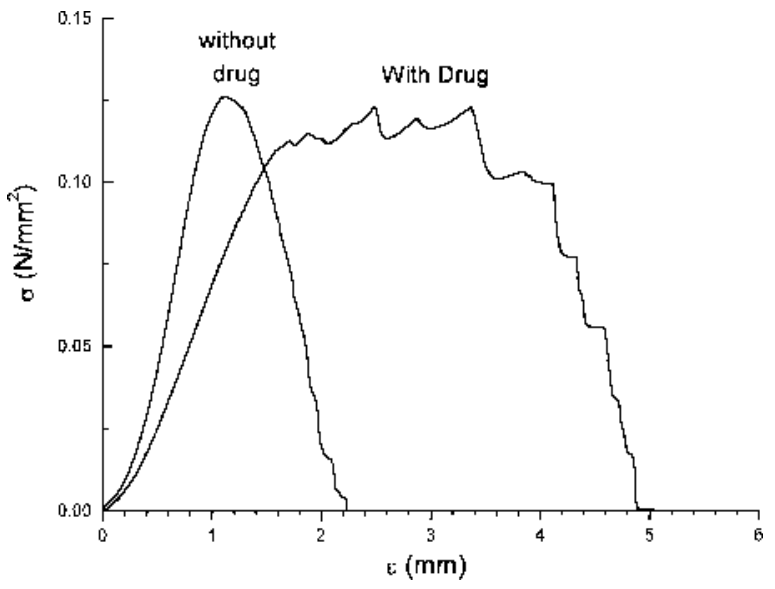

Fig. 9. Force-Elongation Curves for Carbopol 940 Hydrogels with and Without Drug

To evaluate the effect of $\beta \mathrm{CD}$ on adhesiveness, work of adhesion was calculated for hydrogels prepared containing 5 or $20 \% \beta \mathrm{CD}$, under conditions of limited (LHC) and nonlimited (NLHC) hydration. The results obtained (Fig. 10) demonstrate the great importance of hydration conditions.

Considering hydrogels without drug, $\beta \mathrm{CD}$ had opposite effects depending on the degree of hydration of the hydrogel. Under LHC, increasing $\beta$ CD content led to a slight increase in work of adhesion. In other words, $\beta \mathrm{CD}$ has important effects on adhesiveness in relatively dry environments, acting as a wetting agent and permitting the more rapid and effective establishment of adhesive bonds. De Vries et al. ${ }^{19)} \mathrm{ob}-$ served a similar effect in studies of polyacrylic acid films and a surfactant (Brij 96). Under NLHC, by contrast, wetting processes become less important, and the resistance of the swollen gel plays a key role. Under these conditions, the presence of $\beta \mathrm{CD}$ scarcely modified the adhesiveness of crosslinked hydrogels, though some effect was observed in
Table 1. Young's Modulus in MPa (Mean \pm S.D., $n=6$ ) Obtained in the in Vitro Bioadhesive Assays.

\begin{tabular}{|c|c|c|c|c|}
\hline \multirow{2}{*}{ Formulation } & \multicolumn{2}{|c|}{ LHC method } & \multicolumn{2}{|c|}{ NLHC method } \\
\hline & Without drug & With drug & Without drug & With drug \\
\hline \multicolumn{5}{|c|}{ Carbopol $^{\mathbb{R}} 941$} \\
\hline SR & $0.129 \pm 0.013$ & $0.098 \pm 0.006$ & $0.028 \pm 0.014$ & $0.019 \pm 0.005$ \\
\hline $90{ }^{\circ} \mathrm{C}$ & $0.112 \pm 0.007$ & $0.071 \pm 0.004$ & $0.052 \pm 0.070$ & $0.018 \pm 0.005$ \\
\hline $110^{\circ} \mathrm{C}$ & $0.086 \pm 0.010$ & $0.060 \pm 0.005$ & $0.015 \pm 0.010$ & $0.018 \pm 0.006$ \\
\hline \multicolumn{5}{|c|}{ Carbopol $^{\circledR} 934$} \\
\hline SR & $0.121 \pm 0.010$ & $0.083 \pm 0.004$ & $0.022 \pm 0.010$ & $0.020 \pm 0.009$ \\
\hline $90^{\circ} \mathrm{C}$ & $0.125 \pm 0.007$ & $0.065 \pm 0.003$ & $0.024 \pm 0.007$ & $0.010 \pm 0.005$ \\
\hline $110^{\circ} \mathrm{C}$ & $0.083 \pm 0.005$ & $0.066 \pm 0.004$ & - & - \\
\hline \multicolumn{5}{|c|}{ Carbopol $^{\circledR} 940$} \\
\hline SR & $0.111 \pm 0.010$ & $0.083 \pm 0.004$ & $0.032 \pm 0.013$ & $0.009 \pm 0.004$ \\
\hline $90^{\circ} \mathrm{C}$ & $0.105 \pm 0.020$ & $0.065 \pm 0.003$ & $0.017 \pm 0.023$ & $0.009 \pm 0.002$ \\
\hline $110^{\circ} \mathrm{C}$ & $0.072 \pm 0.020$ & $0.066 \pm 0.004$ & - & - \\
\hline
\end{tabular}

Results are shown for hydrogels prepared with or without drug at different crosslinking temperatures $\left(90,110\right.$ or $130^{\circ} \mathrm{C}$, or no crosslinking (NC)).

noncrosslinked hydrogels. The rapid solubilization of $\beta \mathrm{CD}$ within the noncrosslinked network may cause a marked reduction in the gel's mechanical resistance.

Considering drug-containing hydrogels, the inclusion of $\beta C D$ led to a decline in work of adhesion under LHC, and to an increase in work of adhesion under NLHC. Low $\beta C D$ concentrations had opposite effects depending on whether the hydrogel contained or did not contain drug; at high $\beta \mathrm{CD}$ concentrations, however, similar results were obtained for hydrogels with and without drug. This is attributable to inhibition of the formation of the ionic complex between polymer and drug, so that the hydrogel maintains its intrinsic elasticity.

\section{Conclusions}

Carbopol frequently interacts with cationic drugs and ex- 


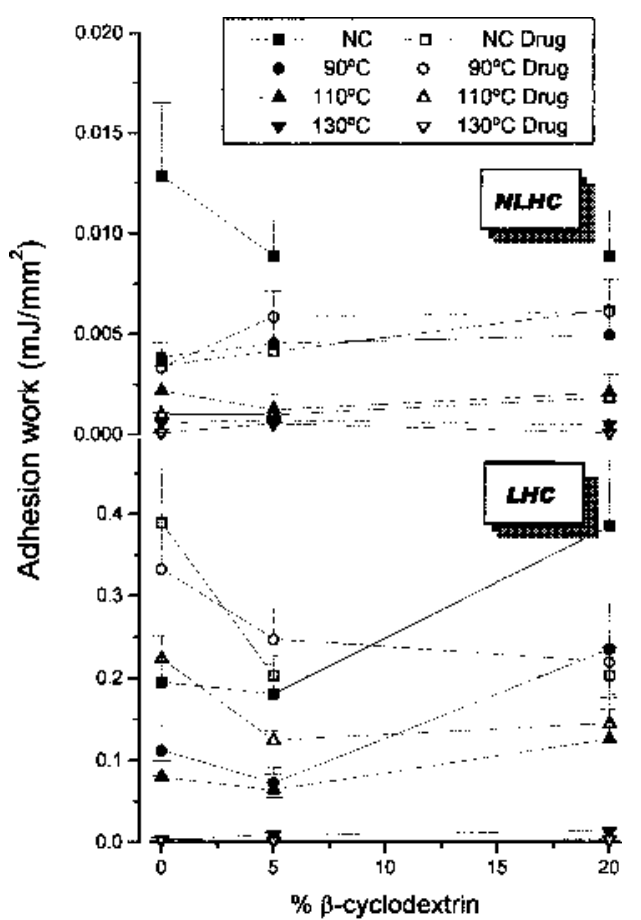

Fig. 10. Plots of Work of Adhesion against $\beta$ CD Content, with Work of Adhesion Assayed as Described in Materials and Methods, under LHC and NLHC Respectively

Results are shown for hydrogels prepared with or without drug at different crosslinking temperatures $\left(90,110\right.$ or $130^{\circ} \mathrm{C}$, or no crosslinking (NC)).

cipients, due to its numerous carboxylic acid groups. Carbopol and PRP hydrochloride interact ionically, giving rise to an insoluble complex whose properties, both in solution and in the solid state, are markedly different from those of the polymer alone. The results of the present study indicate that the interaction affects properties as important as drug release and adhesiveness, with clear repercussions for the efficacy of the dosage form. $\beta \mathrm{CD}$ competes with the polymer for binding sites on the drug, since it is able to form stable inclusion complexes with the drug in solution and in the solid state. The inclusion of propranolol within $\beta \mathrm{CD}$ impedes its interaction with the acid groups of the polymer, thus preventing modification of hydrogel properties. A priori formation of drug/cyclodextrin inclusion complexes is not necessary: it is sufficient for the three components to be mixed simultaneously.

\section{References}

1) Peppas N. A., Korsmeyer R. N., "Hydrogels in Medicine and Pharmacy," Vol. 3, ed. by Peppas N. A., CRC Press Inc., Boca Raton, FL, 1987, pp. 109-135.

2) Grodzinsky A. J., Grimshaw P. E., "Pulset and Self-Regulated Drug Delivery," ed. by Kost J., CRC Press Inc., Boca Raton, FL, 1990, pp. 47-89.

3) Amiji M., Tailor R., Ly M. K., Goreham J., Drug Dev. Ind. Pharm., 23, 575-582 (1997).

4) Huang Y., Leobandung W., Foss A., Peppas N. A., J. Controlled Release, 65, 63-71 (2000).

5) Blanco-Fuente H., Vila-Dorrio B., Anguiano-Igea S., Otero-Espinar F. J., Blanco-Mendez J., Int. J. Pharmaceut, 138, 103-112 (1996).

6) Burgalassi S., Chetoni P., Saettone M. F., Eur. J. Pharm. Biopharm., 42, 385-392 (1996).

7) Le Ray A. M., Iooss P., Gouyette A., Vonarx V., Patrice T., Merle C., Drug Dev. Ind. Pharm., 25, 897-904 (1999).

8) Al Gohary O. M., Foda N. H., Pharm. Ind., 55, 523-527 (1993).

9) Garcia-Gonzalez N., Blanco-Fuente H., Anguiano-Igea S., DelgadoCharro B., Otero-Espinar F. J., Blanco-Mendez J., S.T.P. Pharma, 6, 494-499 (1992).

10) Perez-Marcos B., Ford J. L., Armstrong D. J., Elliott P. N., Rostron C., Hogan J. E., J. Pharm. Sci., 85, 330-334 (1996).

11) Szejtly J., "Cyclodextrin and Their Inclusion Complexes," Akasemiai Kiado, Budapest, 1982.

12) Thuaud N., Sebille B., Deratani A., Lelievre G., J. Chromatogr., 503, 453-458, (1990).

13) Blanco-Fuente H., Anguiano-Igea S., Otero-Espinar F. J., BlancoMendez J., Biomaterials, 17, 1667-1675 (1996).

14) Otero-Espinar F. J., Delgado-Charro B., Anguiano-Igea S., BlancoMendez J., "Data Adquisition an Measurement Techniques," ed. by Muñoz-Ruiz A., Vromans H., Interpharm Press Inc., Buffalo Grove, IL, 1998, Chapter 12, pp. 295-342.

15) Blanco-Fuente H., Anguiano-Igea S., Otero-Espinar F. J., BlancoMendez J., Int. J. Pharmaceut., 142, 169-174 (1996).

16) Giles C. H., Smith D., Huitson A., J. Colloid Interf. Sci., 47, 755-765 (1974).

17) Anlar S., Capan Y., Hincal A., Pharmazie, 48, 285-287 (1993).

18) Ch'ng H. S., Park H., Kelly P., Robinson J. R., J. Pharm. Sci., 74, 399-405 (1985).

19) De Vries M. A., Boddé H. E., Nascimento A., Busscher H. E., Junginger H. E., S.T.P. Pharma, 5, 847-851 (1989). 